\title{
The "P-stitch": a simplified strategy to achieve valvular symmetry during Ozaki Procedure
}

\author{
Sergio Pirola ${ }^{1}$, Giorgio Mastroiacovo ${ }^{2}$, Gianluigi Bisleri ${ }^{3}$, and Gianluca Polvani ${ }^{1}$ \\ ${ }^{1}$ Centro Cardiologico Monzino Istituto di Ricovero e Cura a Carattere Scientifico \\ ${ }^{2}$ Universita degli Studi di Milano Scienze Biologiche \\ ${ }^{3}$ St Michael's Hospital Heart \& Vascular Program
}

December 23, 2021

\begin{abstract}
during Ozaki procedure, relocating a valvular commisure could be technically demanding. This novel technique provides an easy tool to achieve valvular simmetry avoiding commisure shifting.

The "P-stitch": a simplified strategy to achieve valvular symmetry during Ozaki Procedure

Authors: Pirola $\mathrm{S}^{1}$, Mastroiacovo $\mathrm{G}^{2}$, Bisleri $\mathrm{G}^{3}$, Polvani $\mathrm{G}^{1,2}$

\section{Affiliations:}

1 Centro Cardiologico Monzino, Milano,Italy;

2 Department of Cardiovascular Sciences and Community Health, University of Milan, Italy;

3 Division of Cardiac Surgery, St. Michael's Hospital, University of Toronto, ON.
\end{abstract}

Disclosure: none of the authors has conflicts of interest to disclose

All patients treated with this technique have subscribed an informed consent

Corresponding author: Sergio Pirola, MD. Centro Cardiologico Monzino, via Parea 4, 20138 - Milan, Italy.sergio.pirola@ccfm.it

Note : Considering the fact that the article proposes a new technique that is the combination of already well codified and accepted surgical techniques, IRB approval, consent statement and clinical trial registration are not applicable for your study.

\section{Glossary of abbreviations:}

AVD: Aortic valve disease

AVR: aortic valve replacement

AVNeo: Ozaki procedure

Central message: during Ozaki procedure, relocating a valvular commisure could be technically demanding. This novel technique provides an easy tool to achieve valvular simmetry avoiding commisure shifting.

Abbreviated legend of the Central Picture : Preop and postop measurement of the portion of the valve ring treated with this technique 


\section{Introduction}

Ozaki procedure aims to reconstruct patients' aortic valve using autologous pericardium, offering an alternative treatment to classic AVR. Since its first description by Ozaki, AVNeo has yielded promising results in adult populations worldwide, either in presence of bicuspid or tricuspid aortic valve. $[1,2]$.

With respect to the tricuspid phenotype, it has been recommended that if the difference between the sizing of each cusps is more than $2 \mathrm{~mm}$, a new commissure should be created to prevent misalignment between the cusps [3]. However, re-locating a commissure could be technically challenging.

Hereafter we describe our original "P-stich technique" which allows to downsize the intercommisural distance in a tricuspid valve and thereby achieve valvular symmetry during AVNeo avoiding the shifting of a commissure from its natural position.

\section{Surgical technique}

Step 1:

After the excision of the leaflets and a careful and complete annulus decalcification, a single 4-0 Prolene is passed through the nadir of the sinus to be reduced. Pulling this stich toward the center of the ring, the assistant can help the operator exposing the corresponding part of the annulus (figure $1 \mathrm{~A}-\mathrm{B}$ )

Step 2:

The two needles of a double arm 4-0 Prolene (TF or RB-1) are subsequently passed upwards from LVOT to the aortic root through the ring respectively through the dot on the left hand side ("needle 1") and through the dot on the right ("needle 2") (figure $1 \mathrm{C}-\mathrm{D}$ ).

Step 3:

"Needle 1" is then passed downward through the dot on the right an then up again through the dot on the left.

Step 4:

While the assistant gently pulls up the stitch on the nadir, the double loop stitch is tied down. This makes the tissue between the dots to be folded upwards (figure 1E).

Step 5:

Finally, the stitch on the nadir is removed and a new measurement of the intercommissural distance is performed (figure $1 \mathrm{~F}$ ).

In case of a not sufficient reduction or an excessive one, the procedure must be repeated marking the two dots a little bit more distant or closer from the nadir, respectively.

\section{Comment:}

To date, this technique has been used in 30 cases. All patients were undergoing isolated AVNeo procedures. Mean age was 42 y.o. (range 24-60) and $75 \%$ were males. None of the patients had a history of major copathologies or surgical history. Mean height was $174 \mathrm{~cm}$ (range 162-189 cm) and weight was $71 \mathrm{~kg}$ (range 57-85 kg). All patients undergoing AVNeo at our Institute are preoperatively studied with Coronary Computed Tomography (CCT) and Transthoracic Echocardiography (TTE).

In borderline cases (e.g., annulus diameter between 21 and $23 \mathrm{~mm}$ ), the surgeon should consider on a caseby-case basis whether this strategy might be applicable in relation to the patient's body surface or whether a standard commissure repositioning procedure would be more appropriate. In all cases of this series, the procedure led to a reduction of a single size of the leaflet. The downsizing has never been reported as excessive, in a single case the reduction has resulted to be not sufficient and the P-stitch had to be removed and repositioned to achieve the optimal result. The portion of the aortic ring treated with the P-stitch was in 
17 cases the non-coronary sinus, in 3 cases the right coronary sinus, in 8 case the left coronary sinus. In the remaining 2 cases (patients affected by isolated annulus enlargement), treatment was reserved simultaneously to 2 different sinuses (in both cases LCC and NCC) to obtain a configuration of 3 cusps of $35 \mathrm{~mm}$.

Intraoperative echocardiography showed a perfect continence of the valve and excellent aortic gradients (mean gradient $5 \mathrm{mmHg}$, range 2-9) in all cases. There were no conversion to prosthetic aortic valve replacement (figure 2).

The pre-discharge echocardiogram always confirmed the intraoperative findings.

In all cases, no major adverse events occurred during surgery and no early postoperative mortality was reported. All patients underwent a clinical and echocardiographic follow-up at 3 months, with findings which were essentially consistent with the echocardiogram performed at discharge. A mild, not significant, further improvement of aortic gradients was reported in two cases (from mean gradient $3 \mathrm{mmHg}$ to $2 \mathrm{mmHg}$ in one case and from $6 \mathrm{mmHg}$ to $5 \mathrm{mmHg}$ in the other one).

The longer follow up for this series of patients is 1 year. For all patients who underwent echocardiogram at one year after surgery, the results were concordant with those found at the 3-month follow-up.

\section{Conclusion}

This novel technique -represented by what is a simple and easily reproducible annulus plication- in this initial series of patients appears to represent a simple and safe tool in the hands of the surgeon to address cusp size discrepancy during AVNeo while avoiding commissure movement.

This has not only significantly speeded up the exectution of this type of procedure by more experienced surgeons, but has also made the approach to AVNeo easier for surgeons in training.

Commissures shifting remains in use for all those patients whose reduction by a single size of a cusp would not be sufficient to e achieve cusp symmetry (with a discrepancy above $2 \mathrm{~mm}$ ) and also for those patients in whom even a minimal reduction of the valve's functional area could lead to patient-prosthesis mismatch (PPM) [4].

Further validation of this novel approach is needed in order to confirm the initial positive findings in this first series of patients.

\section{References:}

[1] Pirola S, Mastroiacovo G, Arlati FG, Mostardini G, Bonomi A, Penza E, Polvani G. Single Center Five Years' Experience of Ozaki Procedure: Midterm Follow-up. Ann Thorac Surg. 2020 Oct 22:S00034975(20)31730-6. doi: 10.1016/j.athoracsur.2020.08.039. Epub ahead of print. PMID: 33597118.

[2] Ozaki S, Kawase I, Yamashita H, Uchida S, Takatoh M, Kiyohara N. Midterm outcomes after aortic valve neocuspidization with glutaraldehyde-treated autologous pericardium. J Thorac Cardiovasc Surg. 2018 Jun;155(6):2379-2387. doi: 10.1016/j.jtcvs.2018.01.087. Epub 2018 Feb 15. PMID: 29567131.

[3] Ozaki S. Ozaki Procedure: 1,100 patients with up to 12 years of follow-up. Turk Gogus Kalp Damar Cerrahisi Derg. 2019 Oct 23;27(4):454. doi: 10.5606/tgkdc.dergisi.2019.01904. PMID: 32082907; PMCID: PMC7018167.

[4] Pibarot P, Dumesnil JG. Prosthesis-patient mismatch: definition, clinical impact, and prevention. Heart. 2006 Aug;92(8):1022-9. doi: 10.1136/hrt.2005.067363. Epub 2005 Oct 26. PMID: 16251232; PMCID: PMC1861088.

\section{Figures :}

Figure 1: A: cusp measurement wider than $35 \mathrm{~mm}$ sizer.B: a 4-0 Prolene stitch is passed through the nadir of the cusp better exposing the ring. $\boldsymbol{C}$ : two dots are marked $3 \mathrm{~mm}$ besides the nadir. $\boldsymbol{D}$ : the two needles of a double arm 4-0 Prolene are passed from LVOT up through the dots. E: using one of the two needles the 
surgeon passes the same stich through the two dots once again creating a double loop around the nadir and ties the stitch down while the assistant gently pulls up the stitch on the nadir. $\boldsymbol{F}$ : perfect match between the $35 \mathrm{~mm}$ sizer and the reduced intercommisural distance

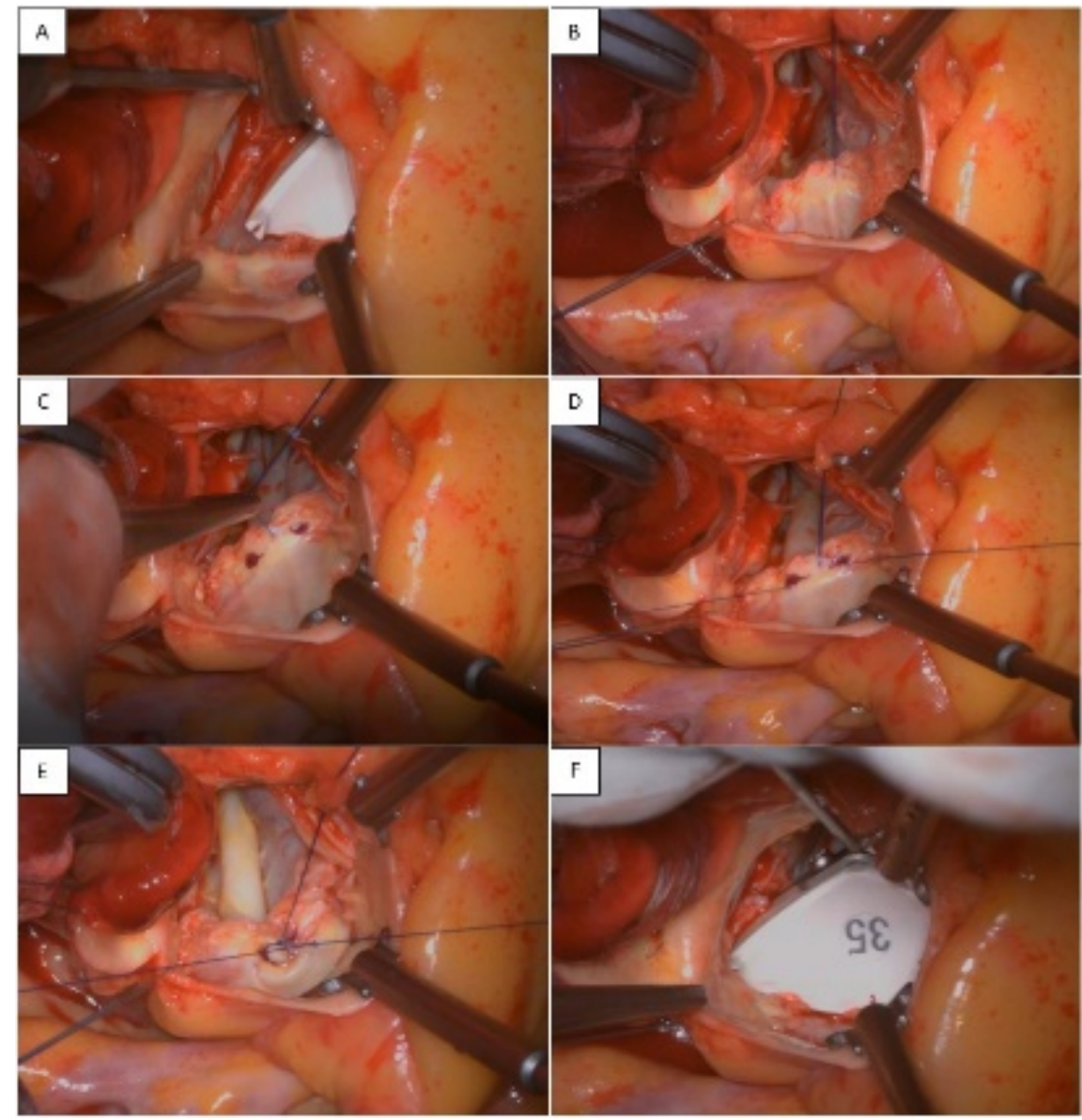

Figure 2: A: 54 y.o. female affected by AS/AR defect. Before and after P-stich. NCC measurements recorded by a certified TEE echocardiographer during telediastolic phase. $\boldsymbol{B}$ : Same patient, peak gradient, mean gradient and transvalvular velocity at the end of surgery 


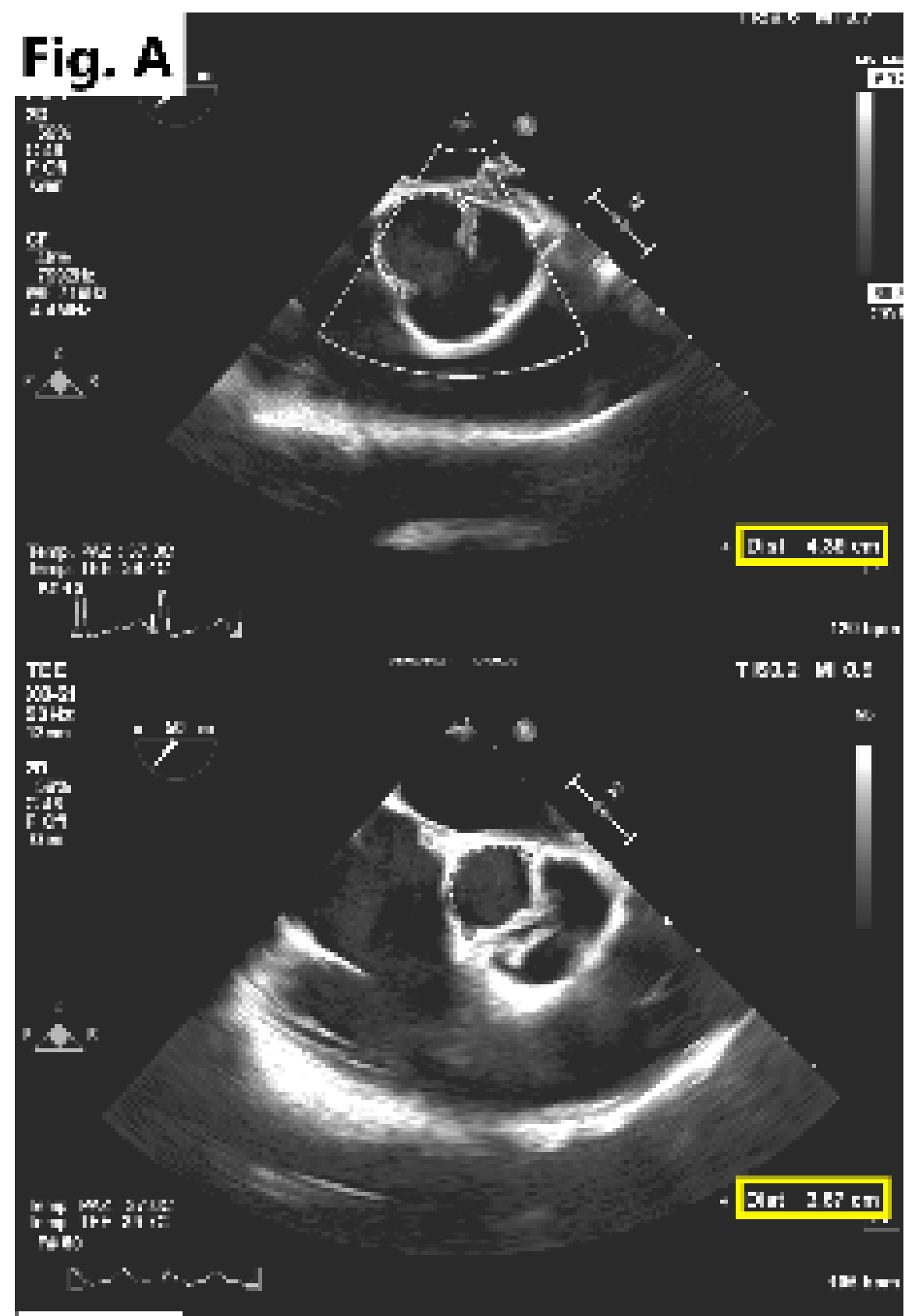

Fig. B

Tas 4 at

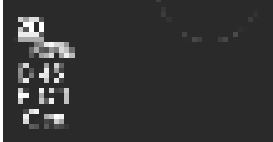

t5

H.

प Yur fy añ Hrits Marm

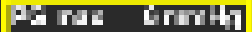
Pintis fint I I IIInI
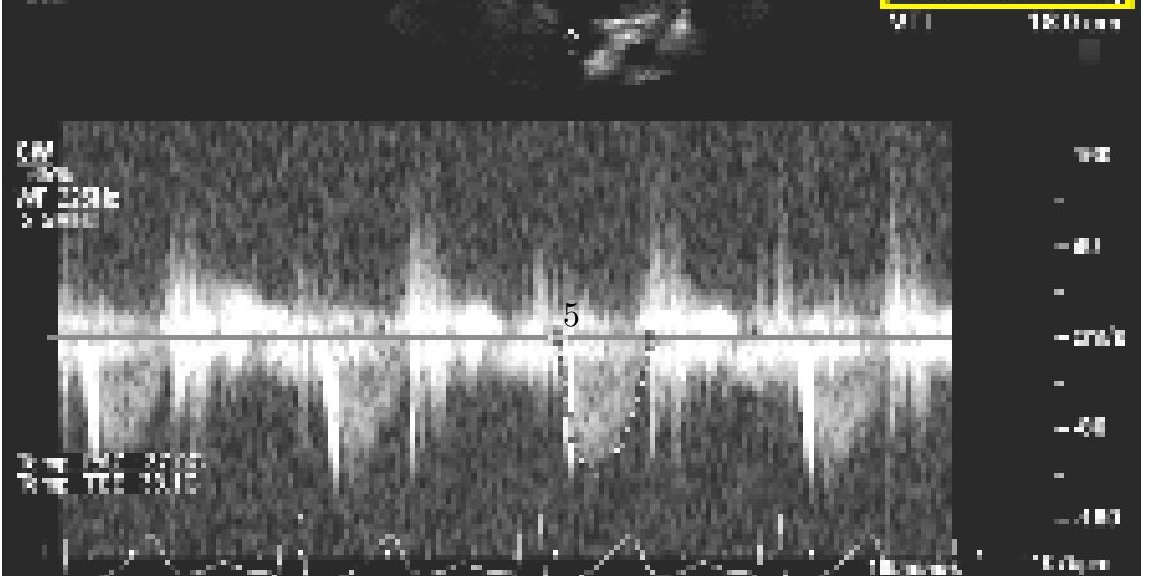
Central figure: 33 y.o male, affected by pure $A R$ and severe annulus dilatation. Virtual $3 D$ measurements (based on CCT) before and after P-stitch on the NCC.
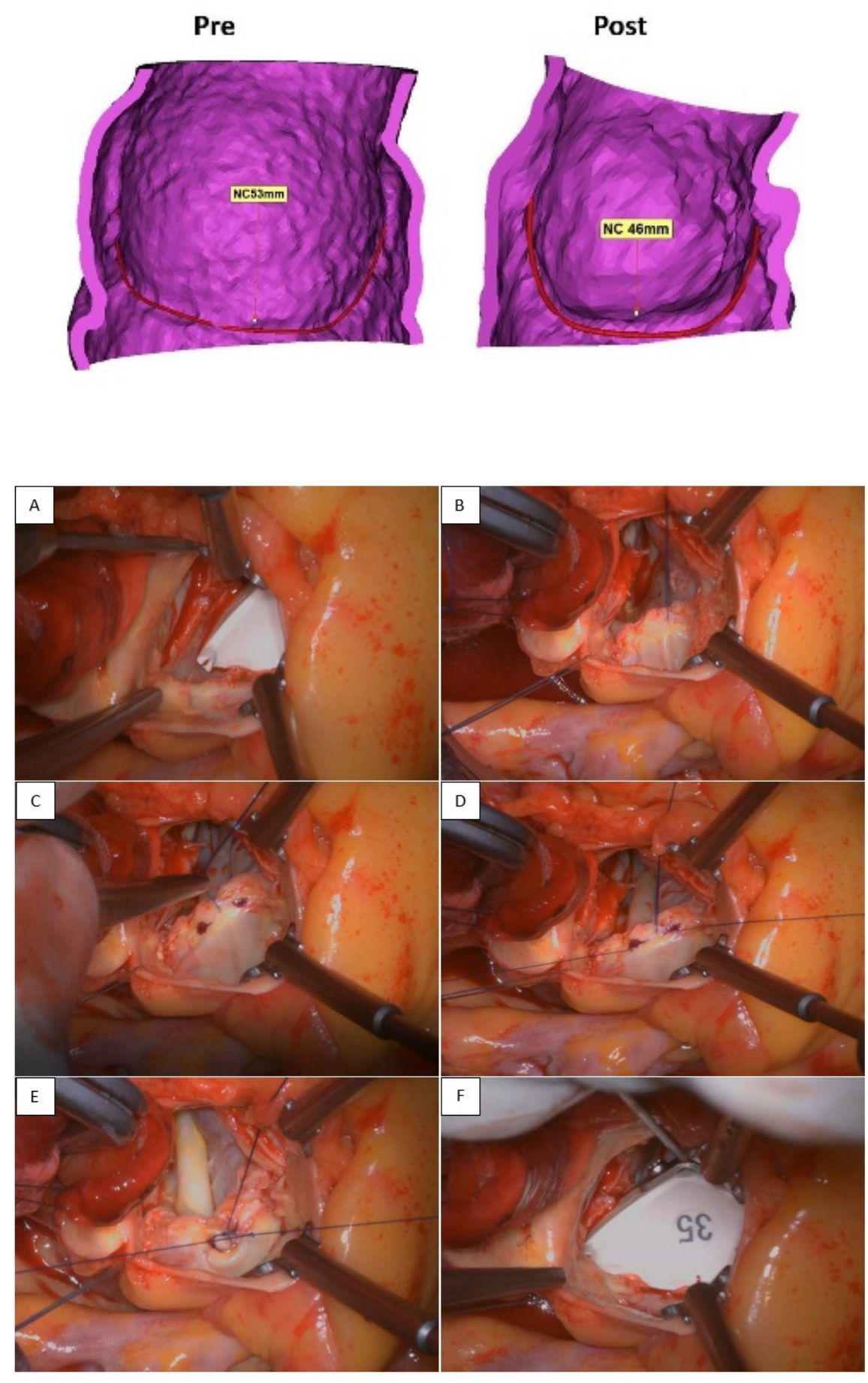
Fig. A
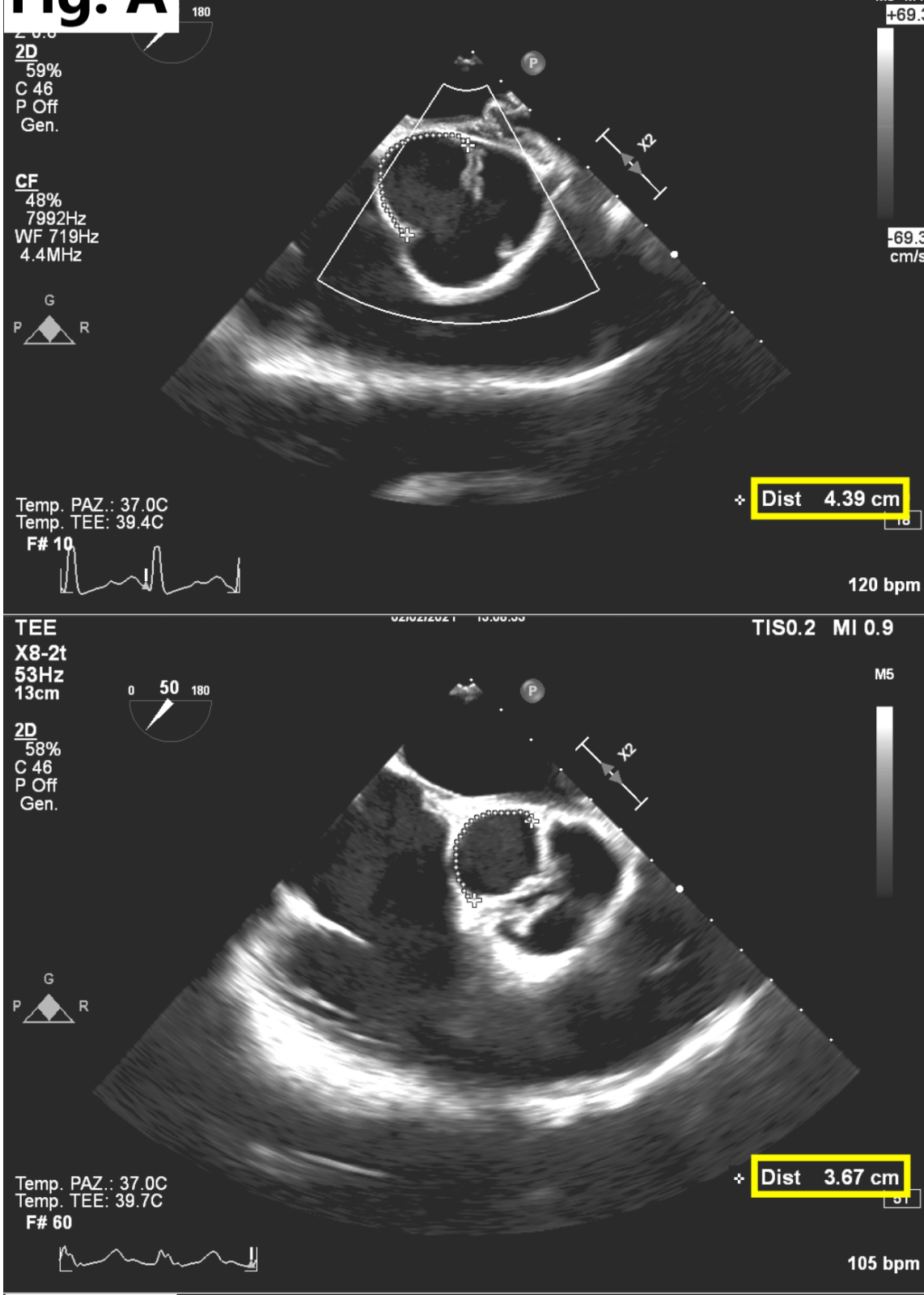

\section{Fig. B}

$15 \mathrm{~cm} \quad 0180$

$\frac{2 \mathrm{D}}{60 \%}$

$60 \%$
c 46

C 46

Gen.

TISO.3 MI 0.0

(P)

M5

+ V max $121 \mathrm{~cm} / \mathrm{s}$

$V$ media $82.6 \mathrm{~cm} / \mathrm{s}$

PG max $6 \mathrm{mmHg}$

PG medio $3 \mathrm{mmHa}$

VTI $\quad 18.0 \mathrm{~cm}$

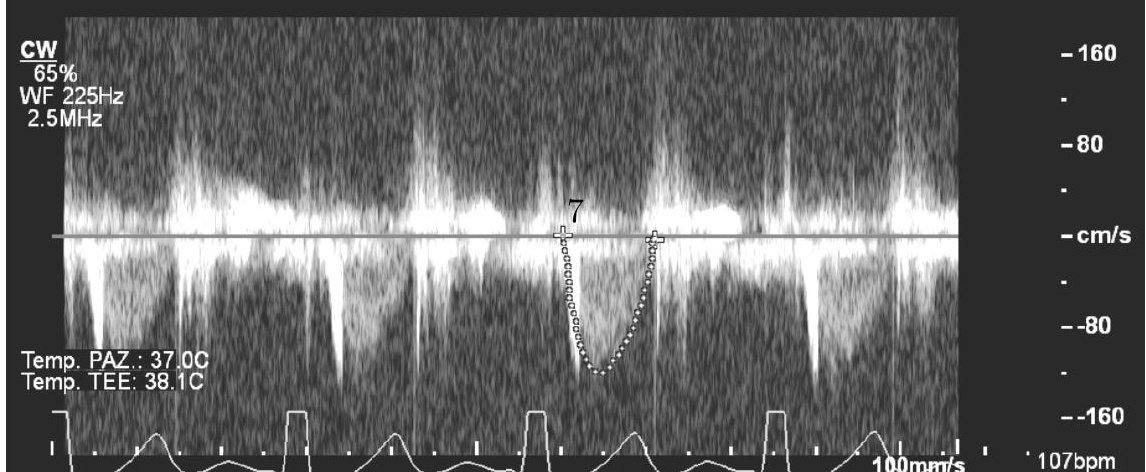



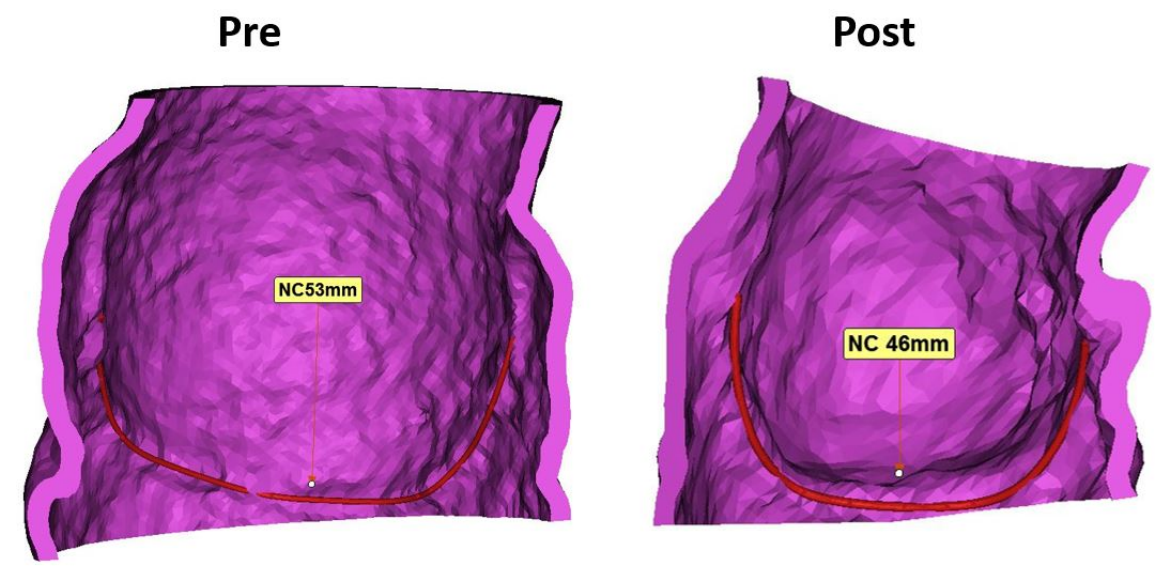